\title{
Nouvelles unités d'enseignement autour de l'étude et de la conception de laboratoires sur puces
}

\author{
Abir REZGUI I, , Morgan MADEC ${ }^{1,2}$, Norbert DUMAS ${ }^{1,2}$, Stephanie GUITON ${ }^{2,4}$, Christophe \\ LALLEMENT $^{1,2}$, Luc HEBRARD ${ }^{2}$, Jacques HAIECH ${ }^{1,3}$, Frederic RUFI ${ }^{4}$ \\ rezgui@unistra.fr \\ ${ }^{1}$ Université de Strasbourg : Telecom Physique Strasbourg, 300 boulevard Sébastien Brandt, 67412 ILLKIRCH \\ 2Laboratoire des sciences de l'Ingénieur, de l'Informatique et de l'Imagerie (ICUBE-UMR7357), boulevard Sébastien Brandt, 67412 \\ STRASBOURG - Pôle CNFM Grand-Est MIGREST \\ ${ }^{3}$ Laboratoire : Laboratoire d'Innovation Thérapeutique (LIT - UMR 7200), Faculté de Pharmacie, 74 rte du Rhin, 67401 ILLKIRCH \\ ${ }^{4}$ Bürkert Fluid Control System - Rue du Giessen, Triembach au Val, France
}

\begin{abstract}
Résumé --- L’article décrit les travaux de recherche d'enseignants-chercheurs de l'équipe "Systèmes et microsystèmes hétérogènes" du laboratoire ICube (Strasbourg) dans le domaine du développement d'outils d'aide à la conception des laboratoires sur puces. Le développement de cette thématique s'est fait en synergie avec l'école d'ingénieur Télécom Physique Strasbourg (TPS), en particulier par le biais d'un nouveau diplôme TICSanté de TPS. Un cursus d'ingénieur en physique avec une forte coloration médecine et biotechnologie. Cette formation leur permet notamment d'acquérir le bagage théorique nécessaire pour le développement d'applications microfluidiques et des laboratoires sur puce. Ils approfondissent leurs compétences également dans des projets autour du développement d'outils de conception associés.
\end{abstract}

\section{INTRODUCTION}

Le concept de laboratoires sur puce (Lab On Chip) est apparu fin des années 90 pour répondre aux besoins d'analyses rapides, sensibles, à haut-débit et à faible coût. Ces laboratoires sur puce s'inscrivent à l'interface entre la biologie et les autres domaines des sciences de l'ingénieur [1]. Ils correspondent à la miniaturisation des systèmes qui rassemblent une ou plusieurs fonctions de laboratoire de plusieurs domaines tels que l'électronique, la thermique, la biochimie ou la microfluidique [2]. Ils visent à réaliser les différentes étapes d'un protocole de mesure impliquant les analyses chimiques et biologiques portant sur l'ADN, les protéines ou les cellules.

Ces microsystèmes ouvrent de nouvelles voies pour l'exploration et la compréhension du vivant en recherche génétique, pour des applications médicales et pharmaceutiques [3]. Ils offrent aussi de nouvelles perspectives dans d'autres domaines tels que la protection de l'environnement [4]. Ces derniers possèdent de multiples applications expliquent l'engouement actuel pour cette thématique de recherche. Cependant, la conception et la fabrication de ces systèmes à l'échelle industrielle reste difficile.

Pour accélérer l'émergence de ces nouvelles applications, l'amélioration des technologies doit s'accompagner du développement de méthodologies et conceptions robustes et des outils associés. Dans ce cadre, le laboratoire ICube (Strasbourg) développe en partenariat avec le Laboratoire d'Innovations Thérapeutiques (Strasbourg) et avec l'entreprise FrancoAllemande Bürkert, une thématique de recherche dans le but d'adresser la problématique de conception des laboratoires sur puce. Notre objectif est de réinvestir nos compétences dans le domaine de la modélisation des systèmes intégrés multi-physique et des technologies de la conception en microélectronique. Plutôt que de développer de nouveaux outils, nous visons à réadapter les outils existants aux spécificités de ces applications nouvelles. L'objectif à terme est de proposer une boîte à outils, intégrant des briques électroniques, fluidiques, biologiques et autres, permettant de concevoir - par prototypage virtuel - un laboratoire sur puce répondant à une spécification donnée. Diverses études préliminaires ont montré le potentiel de cette nouvelle approche et nous ont donc encouragés dans notre démarche [5-8].

Outre le volet recherche, nous avons également mis en place des UE traitant ces problématiques dans le cadre du nouveau diplôme TIC-Santé délivré par Télécom Physique Strasbourg (TPS): une UE de $22 \mathrm{H}$ sur la microfluidique et sa conception en salle blanche et une UE de $60 \mathrm{~h}$ sur la biologie-biochimie en $2 \mathrm{~A}$, et plusieurs UE en $3 \mathrm{~A}(200 \mathrm{H})$ traitant directement ou indirectement des laboratoires sur puce.

Dans cette contribution, nous proposons une présentation des concepts des laboratoires sur puces et leur complexité en matière d'outils de conception liée au caractère multi-domaine. Ensuite, nous détaillerons les objectifs et le fonctionnement de la formation mis en place à Télécom Physique Strasbourg autour des technologies de laboratoires sur puces et nous donnerons quelques exemples de réalisation des étudiants.

\section{LABORATOIRES SUR PUCE}

\subsection{De nouveaux outils pour la biologie}

Le principe des laboratoires sur puces (appelés LOC dans la suite) consiste à faire circuler les réactifs et les échantillons (en volume très réduit), sur différents canaux correspondant aux différentes étapes d'une 
analyse: préparation d'un échantillon, mise en réaction avec différents réactifs, séparation et détection des produits réactionnels [9]. Ces étapes pouvant être séquencés de sorte à former un protocoles expérimental. Il s'agit donc d'un dispositif miniaturisé et intégré dans lequel cohabitent des systèmes de natures différentes comme le montre l'exemple de la Fig. 1: des systèmes de stockage et de transport de fluides (réservoirs, microcanaux, micro-pompes), des systèmes de séparation, des réacteurs biologiques (réaction enzymatique, amplification de l'ADN) ainsi que des modules de détection (micro-capteurs), le tout piloté par un séquenceur électronique.

Ces dispositifs d'analyse miniaturisés à haute intégration disposent de nombreux atouts tels que :

(1) réduire les quantités des réactifs et échantillons,

(2) automatiser, accélérer diverses étapes d'une analyse dans un système unique,

(3) mettre en parallèle les analyses,

(4) combiner les biotechnologies avec des composants électroniques sur une même puce.

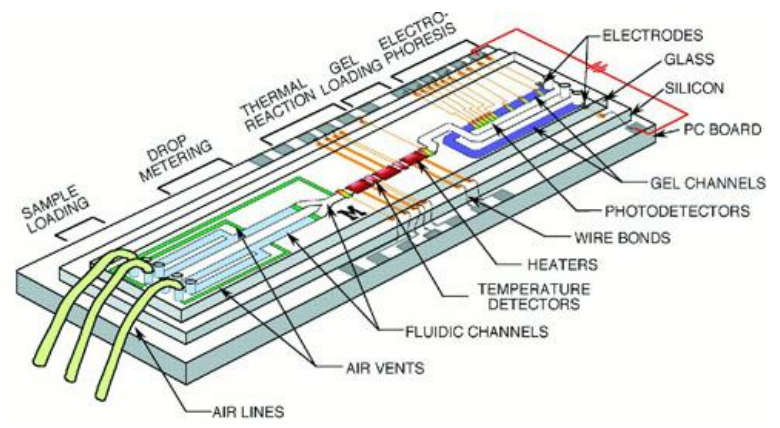

Fig. 1 - Exemple d'un laboratoire sur puce [10]

Les LOC fabriqués sont plus portables. Cette caractéristique est particulièrement intéressante pour le diagnostic à domicile ou pour les urgences sanitaires à grande échelle, dans des zones avec peu d'infrastructures médicales [11].

\subsection{Ce que l'on sait faire ...}

Les premiers travaux novateurs dans ce domaine ont montré la possibilité de miniaturiser des dispositifs d'analyse chimique avec diverses techniques (photolithographie, oxydation et gravure) [12-13] et leurs potentialités. Dès lors, de nombreuses équipes de recherche ont contribué au développement de tels dispositifs, entraînant de nombreuses avancées technologiques [14]. Aujourd'hui, une grande variété de LOC sont développés dans la recherche biomédicale pour l'étude de molécules biologiques (séquençage d'ADN [6], protéines, anticorps, antigènes). Les applications visées sont le diagnostic clinique (virus d'immunodéficience humaine [3], grippe aviaire [15], cancer [16]), la recherche pharmaceutique (criblages haut débit pour la découverte de médicament [17]), et également la protection de l'environnement afin de contrôler la qualité de la nourriture ou la pollution de l'eau (détection de nitrate [5]).

\section{3. ... et ce que l'on voudrait savoir faire.}

Malgré les nombreux efforts, les laboratoires sur puce ayant atteint la maturité nécessaire à leur industrialisation reste rare. La conception et fabrication de ces systèmes reste extrêmement difficile, et donc coûteuse notamment à cause de l'hétérogénéité des phénomènes physiques au sein du même système. Néanmoins, les prévisions d'évolution de ce domaine sont très optimistes. Pour réduire les temps et les coûts de conception et augmenter la fiabilité et les performances es LOC, la mise au point de nouveaux dispositifs portables et éventuellement à usage unique et dont la fabrication se fait à grand échelle devient nécessaire. L'idée serait donc de pouvoir proposer des outils et des méthodes génériques pour leur conception. Ainsi, des efforts supplémentaires autour de la modélisation et la simulation sont nécessaires. C'est dans ce cadre que s'inscrivent les travaux de notre équipe, en collaboration avec l'entreprise Bürkert, dont le but est de concevoir des LOC industriels. Notre objectif est de proposer une boîte à outils (intégrant des briques électroniques, fluidiques, biologiques) permettant de concevoir par prototypage virtuel un LOC répondant à une spécification donnée.

\section{LABORATOIRE SUR PUCE VUE COMME UN SYSTEME HETEROGENE}

\subsection{Exemple de laboratoire sur puce}

Le LOC est par définition un système hétérogène dans la mesure où il combine au moins trois domaines physiques: l'électronique, la biochimie et la fluidique, comme nous allons le voir dans ce premier exemple (Fig. 2). Le LOC étudié est un système de détection de micropolluants dans l'eau potable. Il se compose de quatre sous-systèmes de natures différentes:

(i) un réseau microfluidique qui stocke et transporte des différents réactifs,

(ii) une réaction biochimique produisant des espèces chimiques pouvant être détectées (variation du $\mathrm{pH}$ de la solution),

(iii) un système de détection électrochimique avec l'électronique de conditionnement associé.

(iv) un séquenceur qui commande le système microfluidique et électronique en conformité avec le protocole de mesure.

Le système biologique est basé sur une méthode de dosage immuno-enzymatique (ELISA) compétitif. Ce test est utilisé pour détecter la présence d'une substance, généralement un antigène, dans un échantillon. Il est basé sur une réaction compétitive entre un composé cible, contenu dans l'échantillon à tester, et le même composé couplé à une enzyme et leur reconnaissance par un anticorps spécifique. La séquence du test ELISA est la suivante:

(i) L'échantillon contenant le composé cible $\mathrm{C}$ et celui avec enzyme CE sont introduits dans le réacteur, 
(ii) Pendant l'incubation, une réaction compétitive se produit au cours de laquelle les composés se lient aux anticorps A (déposés sur la surface du capteur). Il y a donc potentiellement moins de CE fixés sur les anticorps A si la quantité de $\mathrm{C}$ dans l'échantillon à tester est grande,

(iii) La chambre est rincée pour évacuer les molécules qui n'ont pas pu se fixer,

(iv) Un substrat $\mathrm{S}$ est ajouté et réagit avec l'enzyme $\mathrm{CE}$ afin de générer un signal mesurable, (variation de $\mathrm{pH})$, provoqué par la réaction enzymatique. Cette variation est d'autant plus rapide que le ratio de complexe CE-A (les CE lié aux anticorps A) est grand, et donc que la concentration en cible $\mathrm{C}$ seul est faible.

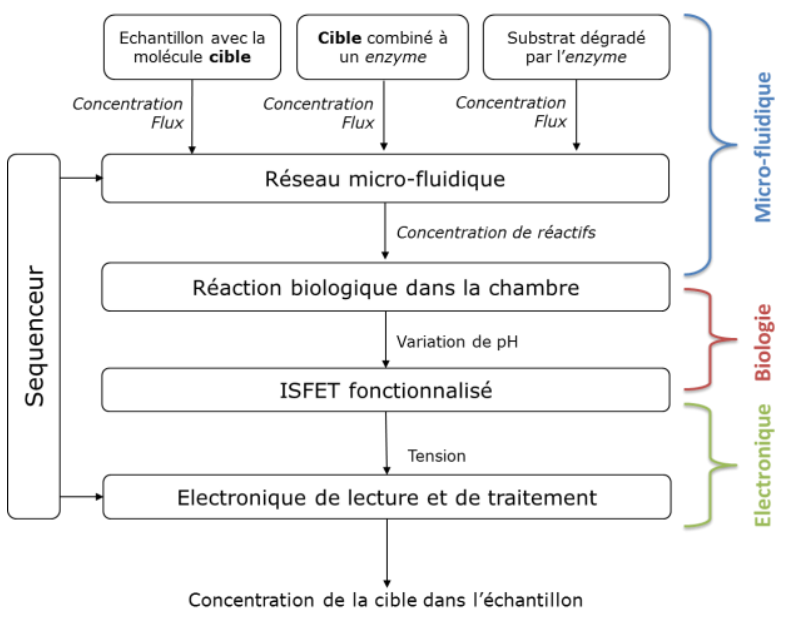

Fig.2 - Décomposition du LOC

Le rôle du circuit microfluidique est l'acheminement des différents réactifs vers la chambre de réaction, à travers un réseau de micro-canaux, en fonction du protocole choisi. Il doit ensuite assurer un mélange homogène des réactifs ainsi que maintenir le mélange dans la chambre pendant la durée d'incubation. Dans l'exemple, trois réservoirs sont nécessaires: un pour l'échantillon $\mathrm{C}$, un pour la molécule cible marquée $\mathrm{CE}$ et un pour le substrat $\mathrm{S}$. Chaque réservoir est couplé à un dispositif de pompage. La chambre de réaction est reliée aux réservoirs de micro-canaux et en contact avec le microcapteur ISFET fonctionnalisé.

Le système de transduction permet de convertir la concentration d'une espèce donnée en une grandeur électrique. Plusieurs approches sont utilisables, pouvant être directes ou indirectes. Dans une détection directe, la composition chimique de la solution modifie les propriétés électriques d'un dispositif. Plusieurs solutions existent: mesure électrique (variation de conductivité), mesure magnétique au travers de GMR (Giant Magnetoresistors) [19], mesure electro-chimiques (variation du $\mathrm{pH}$ mesurée par un ISFET [20]). Pour la mesure indirecte, les informations chimiques sont d'abord récupérées avant d'être mesurées électriquement. Par exemple, la concentration de la cible dans une solution peut modifier ses propriétés optiques (fluorescence), qui sont détectées par un capteur optoélectronique [21]. Les dispositifs mécaniques et les capteurs piézo-électriques peuvent être une alternative pour détecter les espèces biochimiques [22].

Le séquenceur, au final, commande les différentes opérations sur le LOC dans un ordre spécifique selon le protocole de mesure choisi. Il est la plupart du temps un dispositif numérique (microcontrôleur, FPGA ...), avec des interfaces pour les capteurs, actionneurs et autres pièces électroniques du système.

\subsection{Problématique de modélisation d'un LOC}

La modélisation et la simulation des LOC restent difficiles, car ils couvrent différents domaines physiques, chacun d'eux ayant leurs propres outils, langages et formalismes. De plus, les interactions entre les domaines ne sont pas toujours triviales. Des outils de simulation puissants existent dans chaque domaine physique impliqué dans un LOC. Par exemple, le circuit microfluidique peut être modélisé et simulé à l'aide de descriptions numériques, par exemple avec l'outil COMSOL. La méthode éléments finis est le seul moyen disponible pour obtenir des résultats de simulation prédictifs et précis, en prenant en compte des effets comme la tension de surface, un mélange à base de diffusion, etc. Toutefois, cette technique présente deux limites importantes: (1) les simulations peuvent être extrêmement longues et leurs besoins en terme de temps de traitement et de mémoire sont excessifs, (2) la simulation des systèmes fluidiques n'occupe qu'une partie du LOC et il existe un couplage fort entre le comportement de ce sous-système et le reste du système qui n'est pas évident à prendre en compte dans les modèles.

Les modèles analytiques (combinaison d'équations différentielles ordinaires en fonction du temps) sont une alternative. Ces modèles sont moins complexes et donc permettent de gagner du temps de simulation. Le prix à payer est, le plus souvent une réduction de la précision du modèle et donc de sa prédictivité ou une limitation de sa plage de validité. Il s'agit de trouver un compromis entre simplicité du modèle et performances au sens de la qualité des simulations. Une fois validés par comparaison avec les résultats expérimentaux ou des simulations numériques, les modèles analytiques peuvent être assemblés pour former un modèle de niveau système. La qualité et la pertinence de ce modèle au niveau du système dépendent évidemment de la qualité et de la richesse des modèles analytiques élémentaires.

Cette méthodologie est désormais courante pour la conception de systèmes électroniques et de microsystèmes. Les fabricants fournissent de très bons modèles pour la plupart des composants électroniques constituant un circuit. En outre, nous trouvons dans la littérature un certain nombre de travaux d'adaptation de ces méthodologies à des modèles de capteurs et d'actionneurs, tels que par exemple des capteurs optiques ou magnétiques [22]. 


\subsection{Approche proposée}

Cette première analyse a permis d'identifier un certain nombre de verrous à lever dans l'optique de formaliser la conception d'un LOC puce de manière générique. Notre objectif est de développer une boîte à outils de modèles compactes pour chaque partie du dispositif. Nous avons choisi un langage et un environnement de modélisation commun pour les domaines concernés. Le langage VHDL-AMS semble être le candidat le plus approprié, dans la mesure où :

(i) des modèles VHDL-AMS de dispositifs électroniques existent,

(ii) un formalisme pour la description de mécanismes biologiques a été développé par Gendrault et al. [23],

(iii) Voigt et al. ont prouvé qu'il pouvait être adapté aussi pour la fluidique [24],

(iv) son efficacité pour la modélisation multi-domaine au niveau système est prouvée [25].

\section{FORMATIONS ASSOCIÉES}

\subsection{Cadre général}

Avec l'émergence de ces nouvelles technologies et des métiers associés, notamment en lien avec le développement d'approches de $R \& D$, la mise en place des formations spécifiques à ces nouveaux métiers est devenue nécessaire. A ce titre, Télécom Physique Strasbourg est la première école à proposer à ses étudiants une formation sur l'ensemble du cycle ingénieur, spécialisée dans le domaine des TIC appliqués au domaine de la santé (TIC-santé - http://ticsante.ustrasbg.fr). Dans le cadre de cette formation, plusieurs modules ou UE ont été mis en place pour former les étudiants et les préparer aux défis que représentent la mise au point des laboratoires sur puces. Cette formation a pour objectif, notamment, d'acquérir aux étudiants des compétences de base dans les domaines mis en jeu (électronique, fluidique, biochimie) et en méthodologies et outils de conception pour ces systèmes hétérogènes.

Dans cette filière, les étudiants suivent un tronc commun de 4 semestres dans lequel leur sont dispensés des cours d'introduction vers la biologie et les biotechnologies, les biosystèmes et les biocapteurs, les notions fondamentales de la biologie synthétique ainsi que les techniques utilisées pour la mesure et l'observation de phénomènes biologiques (microfluidique, fluorescence etc.). A l'issue de ce tronc commun, les étudiants choisissent entre deux parcours, l'un orienté vers le diagnostic et le traitement médical innovant (parcours DTMI) et l'autre orienté vers les thérapies innovantes (parcours TI). Les futurs ingénieurs ayant choisi le deuxième parcours (TI) sont destinés à être intégrés dans des projets de $R \& D$ multidisciplinaires avec un accent sur les biosystèmes et les technologies

\footnotetext{
${ }^{1}$ http://ticsante.u-

strasbg.fr/doku.php?id=parcours_therapeutiques_innovantes
}

miniaturisées pour la santé dont font bien entendu partie les technologies des laboratoires sur puce. En découle donc des enseignements à la fois multi-physique et multidisciplinaire.

Cette formation, lors du $5^{\mathrm{e}}$ semestre, propose des UE dédiées à la conception de systèmes hétérogènes pour la santé. Étant donné que ces systèmes intègrent des circuits microfluidiques, des réactions biologiques et de l'électronique (comme présenté dans le paragraphe 2), les quatre UE composant ce parcours $\mathrm{TI}^{1}{ }^{1}$ 'intéressent à ces différents aspects.

La première UE "Instrumentation d'analyse \& Système de détection" traite les techniques largement utilisées en détection comme la séparation des cellules par florescence (FACS) ou la détection de liaisons biomoléculaires. Elle aborde aussi l'instrumentation physico-chimique mise en jeu en milieu industriel pour l'élaboration de nouveaux médicaments à but thérapeutique. La deuxième UE "Nano-Sciences et Biosystèmes" familiarise le futur ingénieur avec l'état de l'art de la recherche et des applications biomédicales actuelles. Cette UE s'intéresse aux thèmes relatifs aux nanotechnologies, nanosciences et nanomatériaux utilisés pour les traitements thérapeutiques (par exemple les bio-particules pour le biomédical). Elle traite également des puces ADN et des laboratoires sur puce. $\mathrm{La}$ troisième UE concerne l'Ingénierie Biologique, et propose un aperçu sur les aspects liés à la modélisation et à la conception de systèmes biologiques (biologie synthétique) en s'appuyant sur les méthodologies et l'analogie électronique - biologie [26]. La dernière UE "Conception de Systèmes Intégrés Hétérogènes pour la santé" traite les architectures de systèmes hétérogènes, les méthodologies et le flot de conception au niveau système ainsi que la modélisation et la simulation hautniveau de ces derniers.

Ces diverses UE sont composées d'un certain nombre de cours (micro-fluidique, biologie, électronique, etc.) ainsi qu'un aperçu des applications industrielles présentées par des experts. Ces UE contiennent également de nombreux TP-Projets autour de la modélisation, de la conception et de la simulation avec différents outils, notamment COMSOL et VHDLAMS. Le parcours TI contient un fort volet expérimental avec des TP d'instrumentation optique (plasmonique, FACS, etc.), des TP autour des puces ADN (CIMECNFM de Grenoble) et de la réalisation de circuits micro-fluidiques en PDMS ou gravés sur PMMA ${ }^{2}$ servant à la validation des modèles qui peuvent ensuite être utilisés en recherche.

L'ensemble de ces enseignements est en étroite relation avec des cours autour de la compréhension et la modélisation de réseaux biologiques ainsi que des cours d'électronique intégrée (30h) dans le cadre du Master 2 Micro et Nano Electronique de l'UFR Physique et

${ }^{2}$ La micrograveuse permettant de graver des canaux dans une plaque de PMMA a été financée le CNFM dans le cadre de la mise en place de TP innovants. 
Ingénierie de l'Université de Strasbourg, que les étudiants du parcours TI peuvent suivre en parallèle de la formation TIC-Santé.

En plus de ces UE, un certain nombre de sujets de stage et de projet sont proposés aux étudiants en lien avec nos activités de recherche, en partenariat avec des industriels du secteur. Cette forme de travail permet un bon équilibre entre les recherches amont développées par les enseignants-chercheurs au sein de laboratoire des sciences de l'ingénieur, de l'informatique et de l'imagerie et le développement des outils associés menés par les étudiants. De plus, une certaine synergie se créé entre les étudiants de Télécom Physique Strasbourg et leurs voisins de l'Ecole Supérieure de Biotechnologie de Strasbourg (ESBS) qui apportent également un regard extérieur pertinent à nos travaux.

Nous décrirons dans la suite quelques exemples de TP réalisés pendant la formation. Enfin, dans la partie suivante, un exemple de projet transversal entre l'enseignement et la recherche sera présenté.

\subsection{Fabrication de circuits}

Dans notre formation, nous proposons aux étudiants une journée de TP fabrication de circuits microfluidiques en PDMS dans une salle blanche. Le lieu des TP est la plateforme STnano de Strasbourg qui est une centrale de proximité du réseau RTB. Ces TP sont précédés de trois cours préparatoires sur la microfluidique, les technologies de la salle blanche et la microfluidique appliquée à la biologie.

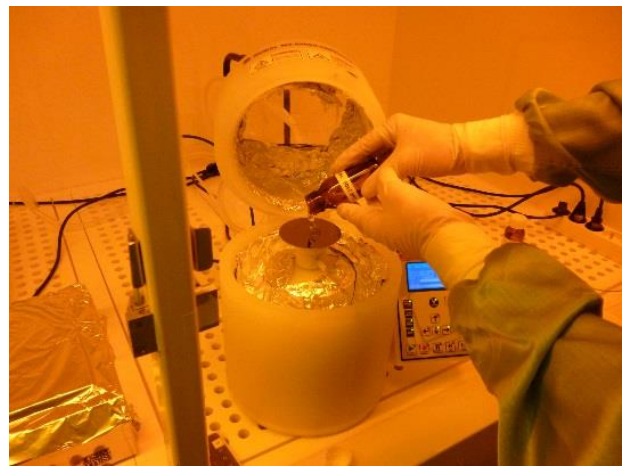

Fig.3 - Tournette dans la zone de photolithographie pour la fabrication du moule en silicium et résine.

L'objectif des TP est de réaliser les étapes de fabrication d'un circuit micro-fluidique. En groupe de 4 à 5 , les étudiants découvrent l'environnement de la salle blanche (Fig. 3) et manipulent leur propre plaquette de silicium au travers des différentes étapes de photolithographie nécessaires à la fabrication d'un moule: dépôt de résine, exposition aux UV et développement du motif en résine. Ensuite du PDMS est coulé sur le moule (Fig.4). Après sa solidification, les étudiants découpent le PDMS, le percent et le collent sur une lame de verre. Le dispositif fabriqué comprend des canaux d'écoulement de quelques dizaines de micromètres.

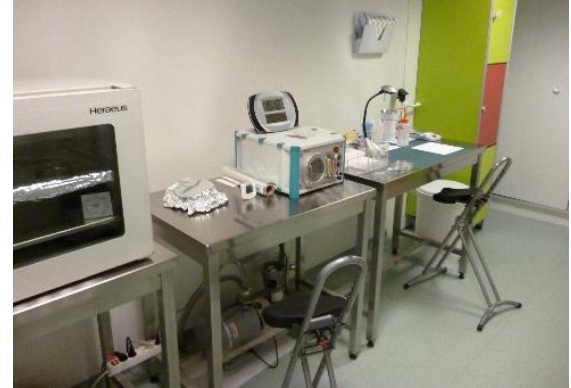

Fig.4-Zone de la salle blanche pour l'assemblage du PDMS. De gauche à droite : étuve à vide, plasma-oxygène et plan de découpe.

Le circuit réalisé est ensuite utilisé pour expérimenter les possibilités de la micro-fluidique en gouttes, notamment étudier la fluorescence de chaque goutte pour le criblage haut débit [26]. C'est l'occasion pour les étudiants d'utiliser le circuit qu'ils ont fabriqué. Après tout le travail préparatoire de connexion du circuit et réglages des pousses seringues, ils observent la fluorescence émise par des fluorophores en goutte par le moyen de photomultiplicateurs PMT. Ils étudient comment varie la taille des gouttes en fonction des débits imposés par les pousses seringues.

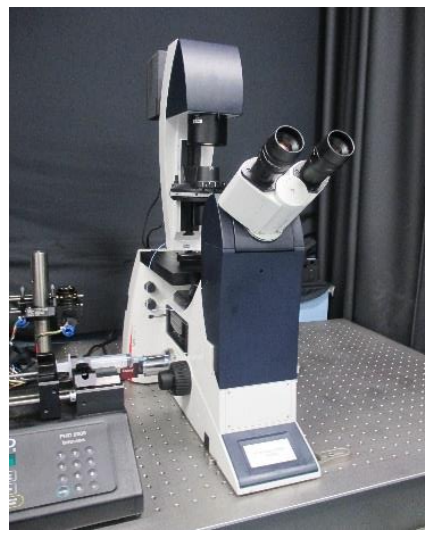

Fig.5-Station microfluidique pour l'expérimentation de la microfluidique en goutte avec microscope, pousse seryngue et banc optique.

Pour la réalisation de projet avec les étudiants de l'école faisant appel à la microfluidique il n'est pas envisageable d'utiliser systématiquement la salle banche pour la réalisation des circuits conçus. L'alternative consiste alors à réaliser des circuits en PMMA par usinage grâce à une machine à commande numérique (Fig.6). Il est alors possible de réaliser des circuits prototypes à bien moindre coût et dans un temps raisonnable.

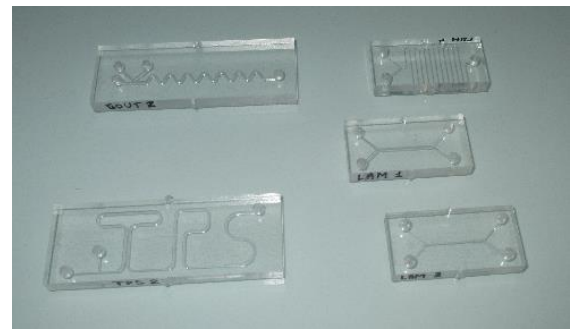

Fig.6-Circuits en PMMA réalisé par micrograveuse à commande numérique 


\subsection{Ecoulements COMSOL}

Cette thématique traite de l'étude des LOC par le biais de la simulation des micro-écoulements continus. L'objectif ici est d'introduire un logiciel de simulation basé sur la méthode des éléments finis et faire ressentir aux étudiants les différences entre écoulements aux échelles macroscopiques et microfluidiques.

Le premier TD est une prise en main de COMSOL avec des généralités sur les écoulements laminaires à travers l'étude d'un mélangeur; basé sur la diffusion des molécules entre les différentes couches lamellaires appartenant aux différents fluides. Le deuxième TD concerne la prise en compte des effets capillaires en microfluidique avec un système de dispense des médicaments; basé sur la diffusion des médicaments dans une goutte d'eau à travers une membrane perméable. Ensuite, un projet de $12 \mathrm{~h}$ est proposé aux étudiants dont l'objectif est d'optimiser un circuit microfluidique d'un laboratoire sur puce. Ils doivent proposer des solutions industrialisables permettant d'assurer le mélange homogène des réactifs pour une meilleure détection de polluant dans l'eau. Un exemple est présenté dans la Fig. 7.

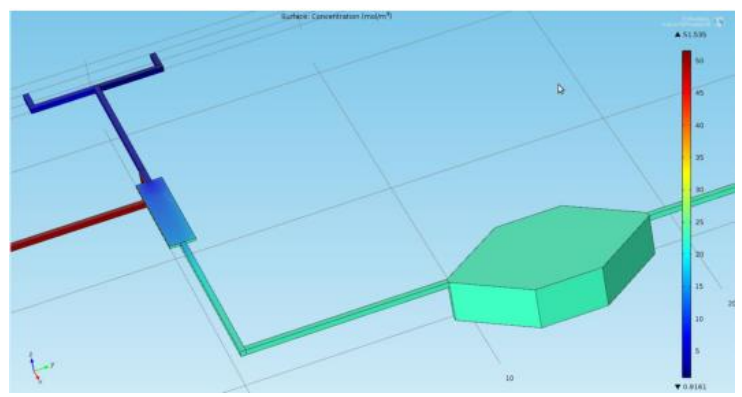

Fig.7- Une solution de mélange basé sur un élément compresseur

\section{EXEMPLES DE REALISATION}

Dans la dernière partie de ce document, nous vous présenterons quelques travaux réalisés par les étudiants dans le cadre de ces formations. Le sujet concerne le prototypage virtuel d'un LOC pour la détection de polluant dans l'eau.

\subsection{Prototypages virtuel d'un laboratoire sur puce pour la détection de polluant en phase aqueuse}

La protection de l'eau est une préoccupation sanitaire majeure et est un sujet de recherche et développement difficile [3]. Avec l'évolution des techniques de traitement et de mesure, les normes deviennent plus rigoureuses et elles prennent en compte de nouveaux composés chimiques comme les micropolluants [3][8]. La détection de ces derniers est complexe, car il est nécessaire de mesurer de nombreuses molécules présentes en faible concentration dans l'eau. L'entreprise Bürkert souhaite exploiter le potentiel des laboratoires sur puce pour atteindre cet objectif. Pour accompagner la réalisation de ce projet, une modélisation haut-niveau et un prototype virtuel du système complet est nécessaire. L'équipe SMH a donc exploré cette voie et proposé des modèles pour les différents circuits microfluidiques, réactions biologiques et micro capteurs en exploitant au maximum le potentiel des langages multiphysiques, notamment VHDL-AMS. La Fig. 8 présente le laboratoire sur puce étudié. Le prototype virtuel de ce système, comme présenté précédemment, contient un circuit microfluidique pour le transport des fluides, une réaction biologique basée sur le kit ELISA et un capteur ISFET mesurant la variation du PH de la solution. Un modèle, de premier niveau, de chacun des sous systèmes a été réalisé et implémenté en VHDL-AMS.

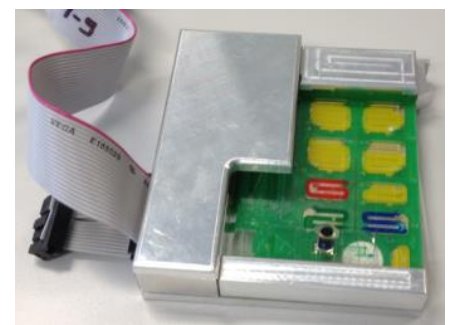

Fig.8-Laboratoire de puce étudié pour l'analyse de l'eau potable

Nous avons identifié, au départ, les composants élémentaires d'un réseau microfluidique (canaux, serpentins, jonctions, ...) et les dispositifs (réservoirs, pompes, vannes, ...). Des premiers modèles ont été construits en se basant sur les lois de Kirchhoff généralisés (conservation de pression et de débit). Les lois de Bernouilli ont été utilisées pour modéliser les réservoirs (Eq. 1) et celles de Navier-Stokes simplifiée pour décrire les canaux en définissant la chute de pression sur la longueur du canal (Eq. 2).

$$
P_{T}+\frac{1}{2} \rho v_{T}^{2}+\rho g z_{T}=P_{C}+\frac{1}{2} \rho v_{C}^{2}+\rho g z_{C}
$$

avec $\rho$ la masse volumique du fluide, g l'accélération de la pesanteur, PT, vT et zT sont respectivement la pression au niveau de la surface, la vitesse du fluide et le niveau de réactif dans le réservoir; et $\mathrm{P}_{\mathrm{C}}, \mathrm{v}_{\mathrm{C}}$ and $\mathrm{zc}$ correspond aux mêmes quantités dans le canal.

$$
\mathrm{Q}=\frac{\Delta \mathrm{P}}{12 \mu \mathrm{L}} \cdot \mathrm{wh}^{3}
$$

où $\mathrm{Q}$ est le débit volumique du fluide, $\Delta \mathrm{P}$ la variation de pression, $\mu$ la viscosité du fluide et $\mathrm{h}$, w et $\mathrm{L}$ sont respectivement la hauteur, largeur et longueur du canal.

Les résultats de simulation VHDL-AMS ont été validés en comparaison par rapport au modèle numérique développé sous COMSOL.

Le système biologique, résumé dans la Fig.9 et décrit en détail dans [18], est un ensemble de réactions chimiques qui provoquent une modification du $\mathrm{pH}$ de la solution, qui est en corrélation avec la concentration du polluant. Les modèles compacts de réactions biologiques sont décrits selon le formalisme développé par Gendrault et al. [24,27]. Chaque réaction a été modélisée avec un module comportant un réseau électrique de composants élémentaires: la résistance (dégradation des espèces chimiques), le condensateur (stockage d'une espèce 
donnée dans un volume donné) et la source de courant commandée en tension (taux d'espèces en fonction de synthèse de la concentration des autres espèces). La structure du modèle proposé est donnée en Fig.10.
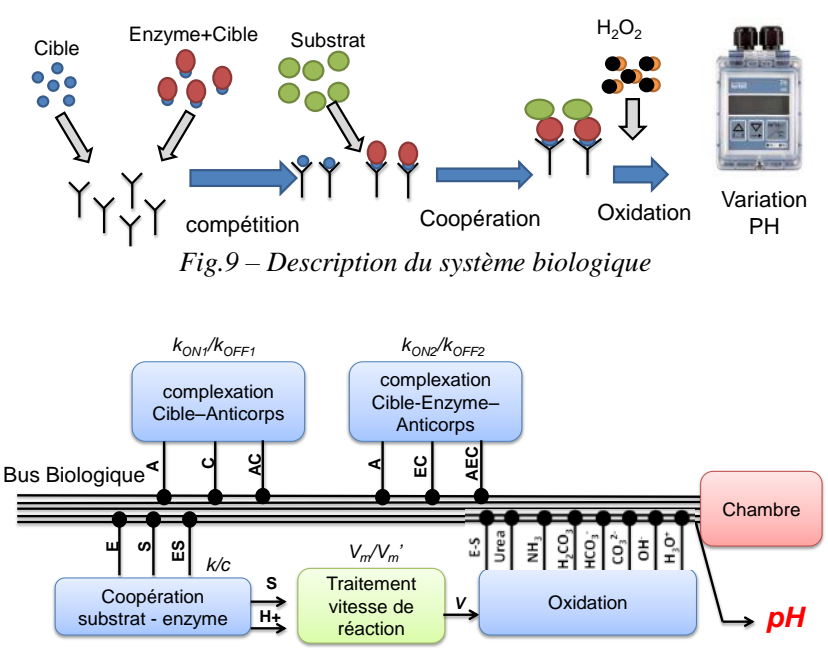

Fig.10 - Modèle du système biologique.

Les deux premiers modules décrivent la réaction de compétition entre la molécule cible $\mathrm{C}$ et la molécule lié à l'enzyme CE pour se fixer sur l'anticorps A:

$\frac{\mathrm{d}[A C]}{d t}=-\frac{\mathrm{d}[C T]}{d t}=k_{O N} \cdot[A] \cdot[C]-k_{O F F} \cdot[A C]$ (Eq. 3)

avec $k_{O N}$ et $k_{O F F}$ les constantes cinétiques de la réaction

Le troisième module correspond à la liaison du substrat $\mathrm{S}$ sur l'enzyme E (A-CE) et le dernier module correspond à la transformation d'un substrat selon Eq. 4. $\mathrm{E}+\mathrm{S} \underset{\mathrm{Kr}}{\stackrel{\mathrm{Kf}}{\Leftrightarrow}} \mathrm{ES} \stackrel{\text { Kcat }}{\longrightarrow} \mathrm{E}+\mathrm{P}$

avec $\mathrm{Kf}, \mathrm{Kr}$ et kcat désignant les différents taux cinétiques.

Dans notre étude, nous avons utilisé la réaction enzymatique d'hydrolyse de l'urée qui provoque une variation du $\mathrm{pH}$ de la solution et qui peut être détectée par un ISFET. Sa cinétique est discutée dans [4].

L'ISFET, quand à lui, est utilisé à un point de fonctionnement statique optimum, correspondant à un courant drain source $\left(I_{D}\right)$ de $100 \mu \mathrm{A}$ et une tension drain source $\left(\mathrm{V}_{\mathrm{DS}}\right)$ de $500 \mathrm{mV}$. Ce point étant fixé, la lecture $\mathrm{du} \mathrm{pH}$ se fait à partir de la tension de grille $\left(\mathrm{V}_{\mathrm{G}}\right)$ (Fig. 11). Des modèles pour l'ISFET et son conditionnement ainsi que l'électronique associé existent déjà.

Dans notre cas, un modèle basé sur l'analogie avec un transistor MOSFET est suffisant en première approximation. La relation, en régime linéaire, entre le courant drain-source $\mathrm{I}_{\mathrm{DS}}$, la tension grille-source $\mathrm{V}_{\mathrm{GS}}$, et la tension drain-source $V_{D S}$ est donnée par l'équation conditionnelle suivante [19]:

$I_{D S}=\beta \cdot\left(V_{G S}-V_{t h}-\frac{1}{2} \cdot V_{D S}\right) \cdot V_{D S}$

avec $\beta$ est un paramètre dépendant de la géométrie et des propriétés physiques de MOSFET, et $\mathrm{V}_{\text {th }}$ est la tension de seuil effective qui dépend du pH selon Eq.6 :

$V_{t h}=V_{T 0}+S \cdot p H$

où VT0 est le décalage introduit par l'analyte à $\mathrm{pH}=0$, et S est la sensibilité de l'ISFET.

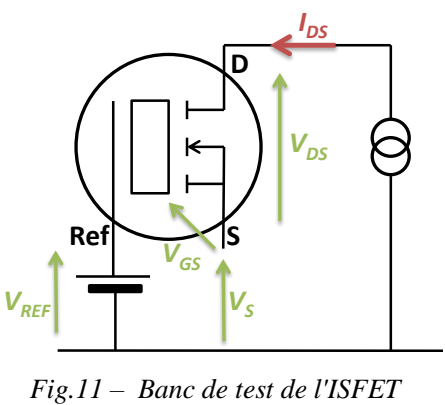

Une série d'expérimentations a été réalisée pour caractériser l'ISFET et ainsi valider le modèle VHDLAMS. La Fig. 12 présente le banc de test.

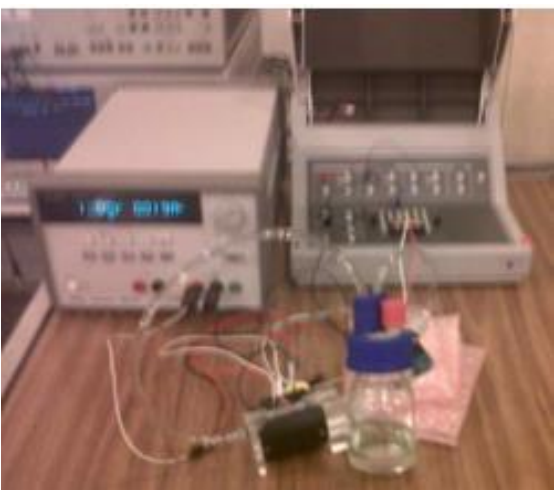

Fig.12 - Banc de caractérisation de l'ISFET

Les résultats sur le courant $I_{D S}$ en fonction de $V_{D S}$ dans la région linéaire et pour une large gamme du $\mathrm{pH}$ sont donnés sur la Fig.13; nous avons un bon accord mesure-simulation. Les données expérimentales conduisent à l'extraction des paramètres du modèle: $\beta=$ $0,305 \mathrm{~mA} / \mathrm{V}^{2}, \mathrm{~V}_{\mathrm{Th}}=2,82 \mathrm{~V}$ et $\mathrm{S}=58,3 \mathrm{mV} / \mathrm{pH}$. Avec ces paramètres, l'erreur quadratique moyenne entre expérimentation et simulation est égale à $1,3 \%$ pour les caractéristiques $I_{D S}-V_{D S}$ et $1,4 \%$ pour les caractéristiques $\mathrm{I}_{\mathrm{DS}}-\mathrm{V}_{\mathrm{GS}}$.

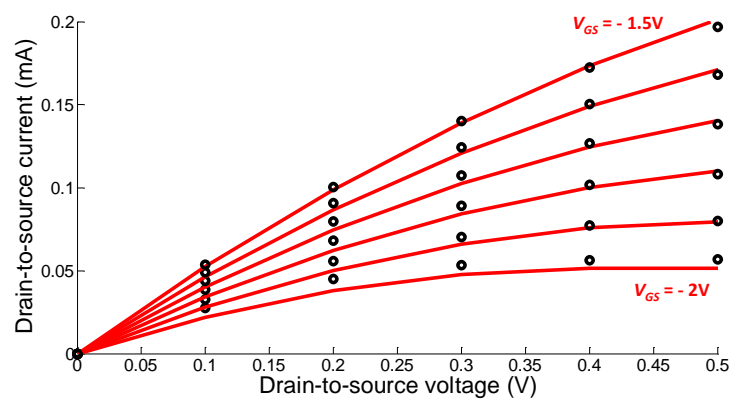

Fig. 13. Caractéristique $\mathrm{I}_{\mathrm{DS}}-\mathrm{V}_{\mathrm{DS}}$ pour différentes $\mathrm{V}_{\mathrm{GS}} \mathrm{à} \mathrm{pH} 7$.

Un modèle de LOC complet, pour détecter des micro-polluants dans l'eau, est proposé en combinant les sous-systèmes présentés en adéquation avec le prototype 
réel présenté en Fig. 6. Ce modèle est utilisé pour suivre la variation du $\mathrm{pH}$ couplé avec le modèle ISFET de voir la tension de sortie pour différentes concentrations du composé cible (Fig.14). Ces travaux ont été publiés lors de la conférence IEEE MIXDES-2014 [18].

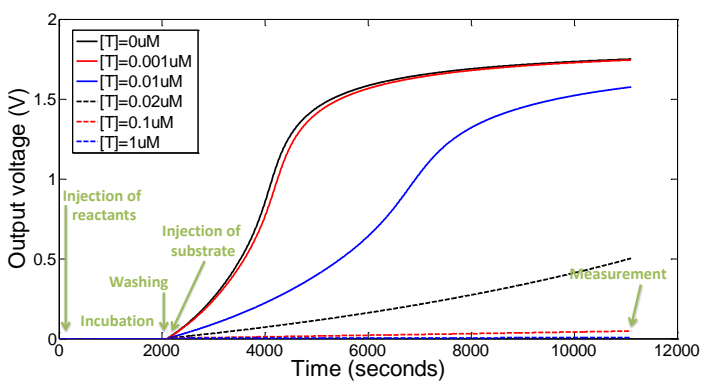

Fig. 14. Tension de sortie pour différentes concentrations de la cible

L'ensemble de module est écrit en VHDL-AMS et simulé sous spectre. Pour le rendre plus accessible et à des fins pédagogiques, le même module a été implémenté sous python et est pilotable au moyen d'une interface graphique (Fig. 15).

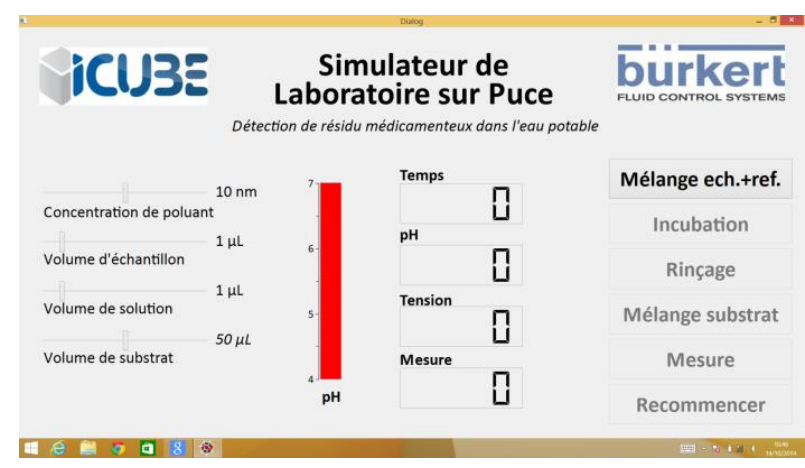

Fig. 15. Simulateur Laboratoire sur Puce

A gauche, l'utilisateur fixe les concentrations de la cible dans l'échantillon, le volume d'échantillon, de la solution et de substrat à injecter. Au centre, des indicateurs permettent de voir le temps et l'évolution du $\mathrm{PH}$, la tension en sortie de l'ISFET et la dernière mesure. A droite, des boutons poussoirs permettent de séquencer la mesure. Au premier temps, la cible et la cible avec enzyme sont mélangés dans les proportions choisies. L'utilisateur clique alors sur "mélange", puis "incubation", puis "rinçage". Après le clic sur "mélange", la modification de paramètres des entrées n'a plus d'effet. Ensuite, il clique sur "mélange substrat". Le module calcule et affiche l'évolution du PH et la tension de sortie minute par minute. "Mesure" permet de figer la valeur en l'inscrivant dans l'indicateur mesure pour la comparer ensuite à un autre set de paramètres.

\subsection{Criblage biologique à base de circuits micro-fluidiques pour la florescence résolue dans le temps}

L'industrie pharmaceutique nécessite des méthodes de test plus avancées, plus rapides et plus efficaces pour identifier les propriétés de molécules bio-actives. La détection de l'intensité de la photoluminescence est largement utilisée dans les applications biotechnologiques à haut débit, notamment pour le criblage à haut débit (HTS) ou la cytométrie en flux (FACS). Grâce à la microfluidique en goutte des milliers de réactions indépendantes peuvent être réalisées par seconde. Dans chaque goutte, les interactions biomoléculaires avec des molécules fluorescentes changent l'intensité lumineuse reçue par des détecteurs optiques. Cette méthode est sensible à la concentration de molécules fluorescentes et à la puissance lumineuse reçue, qui peuvent subir des fluctuations limitant la précision du criblage. Par contre, la durée de vie de la décroissance de fluorescence ne dépend pas de ces paramètres. Elle dépend seulement des interactions à analyser. La mesure de la durée de vie est donc plus fiable. Pour cela une mesure résolue en temps (TSCPC) a été implémentée grâce à des détecteurs de photons uniques à diode avalanche (SPAD). Elles déterminent le temps d'arrivée de chaque photon reçu par rapport à l'impulsion laser (Fig 16.a). Pour des chromophores dont la durée de vie peut descendre à quelques centaines de picosecondes, une résolution de l'ordre de la centaine de picosecondes est nécessaire. Les SPAD sur puce CMOS développées dans l'équipe $\mathrm{SMH}$ répondent à ces spécifications et trouvent donc leur application.

Dans son projet/stage de fin d'étude (PFE) à TPS (stage validé également par le master en Micro et NanoElectronique qu'il suivait également), Jean Pascal Caussé a développé un algorithme de « fit » pour mesurer la durée de vie à partir de l'histogramme des temps d'arrivée des photons (Fig. 16.b). Après comparaison, la méthode du maximum de vraisemblance a été préférée à celle des moindres carrés pour des raisons de précision et aussi de charge de calcul (nombre d'opération arithmétique bien plus faible).
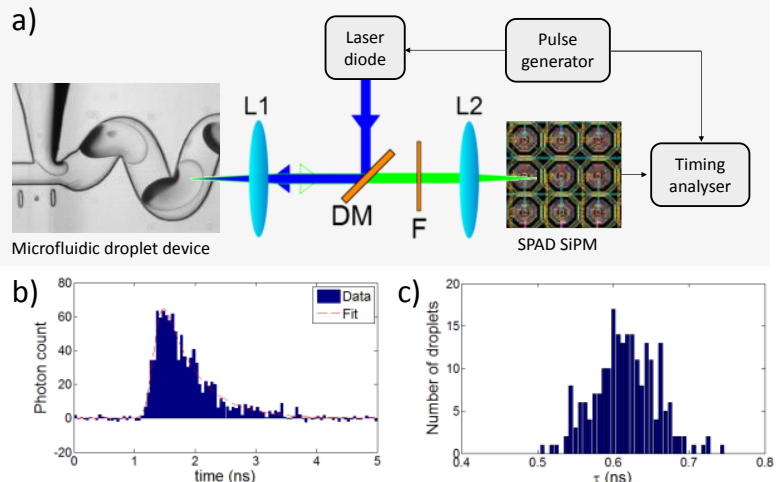

Fig. 16: a) Schéma du banc de test, b) histogrammes du temps d'arrivée des photons après correction, c) histogramme de la durée

de vie mesurée avec de la fluorescéine en présence d'iode.

Ce dernier critère est très important car le haut débit impose une grande rapidité de calcul. Dans la suite du travail de l'étudiant, ces algorithmes sont en cours d'implémentation sur FPGA pour pouvoir traiter la grande quantité de données en temps réel. Comme l'a montré une étude expérimentale et théorique [27], environ 1000 photons de fluorescences peuvent être détectés par goutte de $150 \mathrm{pL}$ avec des concentrations en molécule fluorescente de $300 \mathrm{nM}$, compatible avec l'exigence de ne pas endommager la cible biologique sur laquelle agit la molécule. L'écart type relatif de la mesure 
$(\mathrm{CV})$ est alors suffisamment bon $(<4 \%)$ pour réaliser des criblages biologiques.

La problématique de la focalisation de la lumière sur les gouttes traversant le circuit microfluidique et la collection de la fluorescence a fait l'objet d'études. Un groupe de quatre étudiants de première année de la filière TIC santé ont réalisé un montage à base de composants électroniques et d'une carte Labview qui permet de moduler la lumière d'une diode laser et de démoduler le signal mesuré par des photomultiplicateurs (PMT). Le montage permet de faire des mesures statiques permettant de vérifier la puissance optique reçue par les PMT en fonction de la puissance optique émise. Des études par simulation menées par d'autres étudiants ont permis d'évaluer les performances d'éléments d'optiques intégrées permettant d'optimiser le rapport de la puissance envoyée sur la puissance émise. La puissance d'une diode laser pulsée étant limitée cet aspect est très important, surtout quand la lumière est distribuée sur plusieurs points (multispot) comme il est souhaitable pour paralléliser les analyses ou étudier la cinétique des réactions biomoléculaires.

\section{CONCUSION}

«Le laboratoire sur puce » est donc un nouveau domaine d'étude dont le fort potentiel est reconnu par l'ensemble de la communauté du domaine de la santé et de l'environnement. Ces microsystèmes sont de nature hétérogène, à l'interface des différents domaines physiques comportant des sous-systèmes en Biochimie, en électronique et en microfluidique. Pour être rapidement opérationnels, nous travaillons depuis 2010, en collaboration des industriels et des biologistes, sur des sujets de recherche en amont autour de cette thématique et sensibilisons des ingénieurs, TIC-santé et microélectroniciens de formation, à ces nouvelles problématiques. L'enseignement se fait majoritairement sous la forme de projet et en lien avec nos travaux de recherche, ce qui est bénéfique à la fois pour les étudiants et les encadrants de ces projets.

\section{REMERCIEMENTS}

L'équipe tient à remercier les étudiants de Telecom Physique Strasbourg ayant participé aux différents projets présentés dans cet article : Jean Pascal Caussé (promo 2014), Pauline Zamprogno, Véronique Ferry, Thibaut Wallois (promo 2015) Marc Mounissens (promo 2016).

\section{BIBLIOGRAPHIE}

[1] E. Oosterbroek, A. Van den Berg, "Lab-on-a-Chip Miniaturized Systems for (Bio) Chemical Analysis and Synthesis", 2003

[2] K. E. Herold, "Lab-on-chip technology: fabrication and microfluidics", ed. Horizon Scientific Press, 2009

[3] A. Jang, Z. Zou, K. Kug Lee, C. H. Ahn and P. L. Bishop, "State-of-the-art lab chip sensors for environmental water monitoring", Measurement Science and Technology (2011)
[4] P. Temple Boyer, A. Benyahia, W. Sant, M.L. PourcielGouzy, J. Launay, A. Martinez, "Modeling of ureaEnFETs for haemodialysis applications", Sensors and Actuators (2008)

[5] X. Chang at all, "A microfluidic device for pratical labelfree CD4+ T cell counting of HIV infected subjects", Lab Chip (2007)

[6] A. Habib, F. Pêcheux, "Modeling and simulation of a manycore PCR-CE lab-on-chip for DNA sequencing using SystemC/SystemC-AMS", IEEE International Behavioral Modeling and Simulation Conference, 2010,

[7] A. K. Yetisen, L. Jiang, J.R. Cooper, Y Qin, R. Palaniveluand Y. Zohar, "A microsystem-based assay for studying pollen tube guidance in plant reproduction", Journal of Micromechanics and Microengineering (2011).

[8] I. Humenyuk at all, "Development of pNH4-ISFETS microsensors for water analysis", Microelectronics Journal (2006)

[9] P.-A. Auroux, D. Iossifidis, D.R. Reyes \& A. Manz, « Micro total analysis systems. 2. Analytical standard operations and applications », Anal. Chem., vol. 74, juin 2002

[10] Burns, M.A. et al. "An Integrated Nanoliter DNA Analysis Device," Science, 1998.

[11] P. Yager, T. Edwards, E. Fu, K. Helton, K. Nelson, M.R. Tam et B.H. Weigl: Microfluidic diagnostic technologies for global public health. Nature, July 2006.

[12] A. Manz, D.J. Harrison, E.M.J. Verpoorte, J.C. Fettinger, A. Paulus, H. Lüdi \& H.M. Widmer, «Planar chips technology for miniaturization and integration of separation techniques into monitoring systems - capillary electrophoresis on a chip », J. Chromatogr. A, vol. 593, Feb. 1992

[13] R.D. Oleschuk, L.L. Shultz-Lockyear, Y. Ning et D. J. Harrison: Trapping of bead-based reagents within microfluidic systems: on-chip solid-phase extraction and electrochromatography. Anal. Chem., Feb. 2000

[14] M. U. Kopp, H. J. Crabtree \& A. Manz, « Developments in technology and applications of microsystems ", Current Opinion in Chemical Biology, vol. 1, no 3, oct. 1997

[15] Charlton, B. Crossley \& S. Hietala, « Conventional and future diagnostics for avian influenza », Comparative Immunology, Microbiology \& Infectious Diseases, avr. 2008

[16] B.L. Ziober, M.G. Mauk, E.M. Falls, Z. Chen, A.F. Ziober \& H.H. Bau, «Lab-on-a-chip for oral cancer screening and diagnosis », Head \& Neck, vol. 30, no 1, Jan. 2008

[17] B.H. Weigl, R.L. Bardell \& C.R. Cabrera, «Lab-on-achip for drug development ", Adv. Drug Deliv. Rev., vol. 55, no 3, 2003,

[18] S. Guiton, A. Rezgui, M. Madec, C. Lallement, F. Rufi, J. Haiech, "Modeling and Simulation of a Lab-On-Chip for Micropollutants Detection", 21th International Conference Mixed Design of Integrated Circuits and Systems, 2014

[19] R. Bashir, "BioMEMS state-of-the-art in detection opportunities and prospects", Advanced Drug Delivery Reviews (2004) D.A. Hall et al., "GMR biosensor array: a system perspective", in Biosensors and Bioelectronics, vol. $25,2010$.

[20] P. Bergveld, "Development, operation, and application of the ion-sensitive field-effect transistor as a tool for electrophysiology", IEEE trans. On Biomedical Engineering, vol. 19, 1972

[21] A. Subramanium et al., "Glucose biosensing using an enzyme-coated microcantilever", in Applied Physics Letter, vol. 81, 2002.

[22] J.B. Kammerer, M. Madec, L. Hébrard, "Compact modeling of a magnetic tunnel junction-Part I: dynamic 
magnetization model", in IEEE Trans. Electron Devices, vol. 57(6), 2010.

[23] Y. Gendrault, M. Madec, C. Lallement, J. Haiech, "Modeling biology with HDL languages: a first step toward a Genetic Design Automation tool inspired from microelectronics" Biomedical Engineering, IEEE Transactions on (2014)

[24] P.Voigt, G. Schrag, G. Wachutka, "Microfluidic system modeling using VHDL-AMS and circuit simulation", Microelectronics Journal, 29 (1998)

[25] F. Pecheux, C. Lallement, A. Vachoux, "VHDL-AMS and Verilog-AMS as alternative hardware description languages for efficient modeling of multidiscipline systems", in IEEE Trans. On Computer-Aided Design of Integrated circuits and Systems, , 2005.

[26] M. Madec, et al. "Introduction à la biologie synthétique et au développement d'outils de conception dédiés", CNFM 2012

[27] J. Leonard, Jérémie Léonard, Norbert Dumas, JeanPascal Caussé, Sacha Maillot, Naya Giannakopoulou, Sophie Barre and Wilfried Uhring,"High-throughput time-correlated single photon counting", Lab-on-Chip, vol. 14, Nov. 2014 